Lexis Vol. XLIII (2) 2019: 421-453

\title{
El prohibitivo en las lenguas mayas cholanas y tseltalanas: un estudio microtipológico"
}

\author{
Igor Vinogradov \\ Instituto de Lingüistica, Academia de Ciencias de Rusia \\ Universidad de Bonn
}

\section{RESUMEN}

Este artículo describe la estructura morfosintáctica de construcciones prohibitivas (imperativas negativas) en cinco lenguas mayas del subgrupo cholano-tseltalano. Estas lenguas, aunque son estrechamente emparentadas, utilizan al menos tres distintas estrategias gramaticales para expresar el significado prohibitivo. El análisis corrobora la observación tipológica de que la naturaleza semántica del prohibitivo es más compleja que una simple combinación de la polaridad negativa y la modalidad imperativa.

\footnotetext{
"Este estudio presenta algunos resultados del proyecto de investigación posdoctoral realizado en el Instituto de Investigaciones Antropológicas de la Universidad Nacional Autónoma de México en 2015-2016. Expreso mis agradecimientos al Programa de becas posdoctorales en la UNAM y, en particular, a Cristina Buenrostro por haber asesorado el proyecto. Algunos datos que se discuten en este escrito provienen del trabajo de campo con hablantes nativos. Sinceramente agradezco a todos mis colaboradores. En particular quisiera agradecer a Esmeralda López (chontal) por su apoyo en la organización del trabajo en el estado de Tabasco, México, y a William Marcos García (ch'orti’), en el departamento de Chiquimula en Guatemala. El análisis fue concluido durante la estancia de investigación en la Universidad de Bonn (Alemania) con el apoyo de la Fundación Alexander von Humboldt.
} 
La variación se estudia bajo el enfoque "microtipológico" que, en este caso particular, es superior a un estudio "macrotipológico" basado en un amplio muestreo de lenguas, ya que permite obtener una perspectiva más detallada y trazar hipótesis sobre posibles cambios diacrónicos.

Palabras clave: prohibitivo, lenguas mayas, tipología lingüística, morfosintaxis

\section{ABSTRACT}

This article describes the morphosyntactic structure of prohibitive (negative imperative) constructions in five Mayan languages of the Cholan-Tseltalan subgroup. These closely related languages use at least three different grammatical strategies to express the prohibitive meaning. The analysis corroborates the typological observation that the semantic nature of the prohibitive is more complex than a simple combination of the negative polarity and the imperative modality. The variation is studied under the "micro-typological" approach that, in this particular case, is superior to a "macro-typological" study based on a wide sampling of languages since it allows obtaining a more detailed perspective and plotting hypotheses about possible diachronic changes.

Keywords: prohibitive, Mayan languages, linguistic typology, morphosyntax

\section{Introducción}

Este artículo describe la estructura morfosintáctica de construcciones prohibitivas en cinco lenguas mayas del subgrupo cholano-tseltalano: chol, chontal de Tabasco, ch'orti', tseltal y tsotsil. Estas lenguas llaman la atención particular gracias a una gran diversidad de estrategias gramaticales que utilizan para expresar el significado prohibitivo; véase Vinogradov (2016). Esta variación se analiza bajo el enfoque microtipológico (o "intragenético") que compara lenguas emparentadas (Greenberg 1969; Kibrik 1998).

Este trabajo tiene dos objetivos principales. Primero, hacer una descripción de construcciones prohibitivas en las lenguas cholanotseltalanas, analizando las diferencias que se dan entre el prohibitivo 
y el imperativo (positivo), por un lado, y entre el prohibitivo y el declarativo negativo, por el otro. De tal manera, el artículo contribuye a la descripción lingüística de las lenguas mayas del subgrupo cholano-tseltalano. El otro objetivo es metodológico. Aplicando el enfoque microtipológico al estudio de construcciones prohibitivas en un subgrupo particular de la familia maya, se demuestran sus ventajas metodológicas, ya que permite refinar el análisis lingüístico y proponer hipótesis acerca del desarrollo diacrónico.

El artículo está organizado de la siguiente manera. La sección 1 introduce las nociones básicas y aclara el objeto de estudio y la metodología que se utiliza. Las secciones 2 y 3 describen construcciones prohibitivas y sus diferencias de construcciones imperativas y declarativas negativas en tres lenguas cholanas (chol, chontal de Tabasco y ch'orti') y en dos lenguas tseltalanas (tseltal y tsotsil), respectivamente. La sección 4 ofrece una breve discusión sobre el ch'olti', otra lengua cholana, ahora extinta pero documentada durante la época colonial. La sección 5 presenta conclusiones que se derivan del presente estudio.

\subsection{El prohibitivo: un acercamiento macrotipológico}

El prohibitivo es un significado modal, opuesto al imperativo con respecto a la polaridad. Es decir, el prohibitivo equivale semánticamente al imperativo negativo. En vez de expresar una orden o una petición, comunica una prohibición que el hablante quiere imponer al oyente. En otras palabras, el prohibitivo es una de las posibles modalidades de enunciación que tiene por objetivo prohibir que el oyente realice cierta acción.

Los estudios tipológicos han demostrado que en las lenguas del mundo existen varias estrategias para expresar el significado prohibitivo (Aikhenvald 2010: 165-192; van der Auwera et al. 2005). La estrategia más común que se observa en la mayoría de las lenguas es reservar un medio morfológico o léxico dedicado únicamente para denotar prohibición y que no se utiliza en oraciones negativas declarativas y tampoco en imperativas positivas (Miestamo y van der Auwera 2007; van der Auwera 2006). Un ejemplo del latín 
ilustra esta estrategia. ${ }^{1}$ Se emplea el elemento auxiliar noli que se gramaticalizó de la forma imperativa del verbo nolle 'no querer', más el infinitivo del verbo principal (1). ${ }^{2}$

Latín

(1) noli me timere

PROH 1sG.ACC temer.INF

¡No me temas!'

Es posible hacer una clasificación más detallada de marcadores del prohibitivo como noli en (1) según su origen diacrónico o según su lugar en el sistema gramatical. En una lengua individual, el marcador del prohibitivo puede ser parte del lexicón si es una partícula negativa específica o bien ser un morfema gramatical, que forma parte del paradigma de modo junto con los marcadores del imperativo y del indicativo, por ejemplo.

Si la lengua no tiene medios específicos para expresar el significado prohibitivo, puede, en general, seguir dos escenarios (van der Auwera 2010): 1) negar la forma imperativa o 2) negar la forma declarativa, ya sea en el modo indicativo o subjuntivo/optativo si este existe en la lengua particular. La primera opción se ilustra con los ejemplos del ruso en (2).

\footnotetext{
1 Los ejemplos (1) y (2) son conocimiento propio del autor. Los ejemplos de (3) en adelante provienen del trabajo de campo del autor siempre y cuando no se indique la fuente explícitamente. Si la fuente proporciona una traducción en inglés, la versión en español es del autor.

2 La lista de abreviaciones: 1 - primera persona, 2 - segunda persona, 3 - tercera persona, A - juego pronominal A, ACC - acusativo, AFIRM - afirmativo, APPL - aplicativo, ATTR - atributivo, B - juego pronominal B, C - juego pronominal C, CAUS - causativo, COM - completivo, DEF - artículo definido, DEM - demostrativo, ENCL - enclítico, EXH - exhortativo, EXIST - existencial, FUT - futuro, ILL - ilocutivo, IMP - imperativo, INC incompletivo, INCL - inclusivo, INF - infinitivo, LOC - locativo, MP - mediopasivo, NEG - negativo, NMLZ - nominalización, NUM - numeral, PASS - pasivo, PL - plural, POSs posesivo, PREP - preposición, PROG - progresivo, PROH - prohibitivo, QUOT - citativo, REAL - estatus real, REFL - reflexivo, SG - singular, SUBJ - subjuntivo, sUF - sufijo verbal.
} 
Ruso

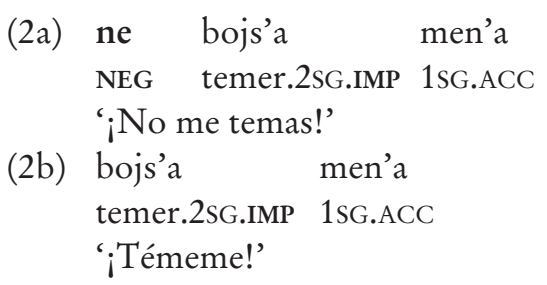

La construcción prohibitiva en el ruso (2a) consta de la forma imperativa del verbo más la negación regular ne. El prohibitivo en ruso es simétrico al imperativo, ya que la única diferencia entre (2a) y (2b) concierne el morfema negativo; véase Miestamo y van der Auwera (2007).

El idioma español presenta el ejemplo de la otra estrategia. Las formas del modo imperativo no se utilizan en las construcciones prohibitivas en español, sino que la negación se añade a las formas del modo subjuntivo, como en No me temas, por ejemplo. El prohibitivo y el imperativo en español son asimétricos: compárese con ¡Témeme! Esta estrategia necesariamente lleva a una ambigüedad. Las formas subjuntivas negadas que se emplean para formar el prohibitivo aparecen también en contextos declarativos negativos desempeñando otra función. Por ejemplo, la misma construcción morfosintáctica en Voy a sonreir para que no me temas no indica el prohibitivo, sino expresa el propósito en la cláusula dependiente.

\subsection{El prohibitivo en las lenguas mayas}

En cuanto a las lenguas de la familia maya, el prohibitivo no ha recibido una atención particular, solo esporádicamente apareciendo en vocabularios y gramáticas de referencia. Esto puede deberse a que en la mayoría de las lenguas mayas el prohibitivo se encuentra en la periferia del sistema de lenguaje entre la gramática y el léxico. Las lenguas del subgrupo k’icheano presentan una excepción remarcable dado que expresan el significado prohibitivo dentro del paradigma gramatical de tiempo/aspecto/modo como lo demuestra el ejemplo de la lengua poqomchi’ en (3). 
Poqomchi'

(3) m-at-joht-ik naah chee' wilih r-uum yohb'aal PROH-2B-subir-REAL sobre árbol DEM 3SG.POSS-causa peligroso 'No subas a este árbol porque es peligroso.'

El prefijo verbal $m$ - indica el prohibitivo en poqomchi'; un negador adicional ya no se necesita. Este morfema está en la distribución complementaria con otros marcadores del mismo paradigma de tiempo/aspecto/modo. En (4), el prefijo $x$ - que indica el aspecto completivo se utiliza en lugar del morfema $m$-.

Poqomchi'
(4) $\mathbf{x}$-at-joht-ik naah chee' wilih COM-2B-subir-REAL sobre árbol DEM
'Subiste a este árbol.'

Para negar la forma completiva, se requiere de un negador que se agrega a la forma verbal sin que el marcador de tiempo/aspecto/ modo se pierda (5). Este negador está compuesto por la partícula prepositiva y el enclítico de modalidad “irrealis"; véase más detalles en Romero (2012), por ejemplo.

Poqomchi'
(5) ma' $x$-at-joht-ik=ta
naah chee' wilih
NEG COM-2B-subir-REAL=IRR sobre árbol DEM
'No subiste a este árbol.'

Cabe decir que el imperativo positivo en el poqomchi' no forma parte del mismo paradigma y se expresa de manera distinta (6).

Poqomchi'
(6) joht-een naah chee' wilih subir-IMP sobre árbol DEM
'Sube a este árbol.'

Las bases intransitivas como joht- 'subir' toman en el imperativo un sufijo especial. Otras clases de verbos exhiben distintas maneras 
de formar imperativos en poqomchi’ (Mó Isém 2006: 188-190). A diferencia de las formas prohibitivas, la forma verbal con el sufijo imperativo -een no lleva el prefijo de persona; compárese (3) y (6). Esta diferencia estructural entre el imperativo positivo y el prohibitivo también se observa en las lenguas cholanas y tseltalanas.

Las lenguas del subgrupo cholano-tseltalano se distinguen considerablemente del poqomchi' y de las lenguas k'icheanas en general, ya que carecen de un marcador dentro del paradigma gramatical de tiempo/aspecto/modo que esté dedicado específicamente a denotar el prohibitivo. Sin embargo, disponen de otros medios tanto léxicos como gramaticales, y emplean varias estrategias de las mencionadas en la sección 1.1. Esta peculiar heterogeneidad hace un estudio comparativo particularmente importante.

\subsection{El método de microtipología}

Un estudio "microtipológico" o “intragenético" se basa en el análisis comparativo de lenguas emparentadas que pertenecen a la misma familia lingüística o a un subgrupo dentro de la familia (Kibrik 1998; Bisang 2004). A menudo estas lenguas también se relacionan geográficamente pues son habladas en la misma región. Los estudios de este tipo recientemente han ganado ciertos privilegios en la tipología lingüística, ya que permiten acceder a la variación que se da dentro de una familia particular vinculando los métodos y objetivos de la tipología con los de la lingüística histórica — de ahí que las diferencias que se observan en las lenguas emparentadas tienen una explicación diacrónica- Pueden ser causadas por contactos, tanto internos como externos, o por las tendencias del desarrollo individual (Shluinsky 2017: 335). Últimamente, varios fenómenos lingüísticos en varias familias del mundo han sido analizados bajo la perspectiva microtipológica; véase, solo por citar algunos ejemplos, Chamoreau (2012), Forker (2013), von Waldenfels (2014) y Shluinsky (2017). El presente artículo continúa desarrollando el mismo enfoque metodológico.

Un estudio microtipológico normalmente se realiza a partir de los datos que provienen de todas las lenguas estudiadas o de la gran mayoría de ellas. El subgrupo cholano-tseltalano, por ejemplo, 
incluye cinco lenguas, y todas están relativamente bien documentadas. Por lo tanto, el presente estudio toma en consideración los datos de todas estas lenguas. Los principios de elección de material oponen el método microtipológico al macrotipológico, ya que el último se funda en un amplio muestreo de lenguas de distintas familias y distintas áreas lingüísticas. Las cuestiones de equilibrio y representatividad del muestreo tan importantes para estudios macrotipológicos no son relevantes para estudios microtipológicos. El muestreo para un análisis macrotipológico a menudo incluye solamente una o dos lenguas más estudiadas de la familia. Por ejemplo, el estudio macrotipológico (van der Auwera y Devos 2012) sobre el uso de la categoría de irrealis en imperativos y prohibitivos se basa en un muestreo de 179 lenguas del mundo que incluye solamente una lengua de la familia maya: el mam. No obstante, sin importar cuál es el fenómeno particular que se estudia, es evidente que las lenguas de la misma familia pueden exhibir una gran variación interna. Esta variación hace que estudios macrotipológicos no sean de todo confiables, ya que los muestreos no representan bien toda la diversidad lingüística.

A pesar de que los dos enfoques - microtipológico y macrotipológico - utilizan los mismos instrumentos comparativos y a menudo derivan sus resultados de un análisis estadístico, no son opuestos uno a otro; considérese también Polinsky (2010). Los dos enfoques persiguen objetivos bien distintos y, en realidad, se aplican en distintas áreas de conocimiento lingüístico; véase Kibrik (1998) para las ventajas y desventajas que tiene cada uno de estos enfoques. Un estudio microtipológico puede servir como la etapa previa a un estudio macrotipológico a fin de equilibrar el muestreo de lenguas y hacerlo más representativo.

\section{El significado prohibitivo en las lenguas cholanas}

Hoy en día, el subgrupo cholano de la familia maya incluye tres lenguas, aunque estudios históricos reconstruyen una mayor variedad lingüística en la época colonial y precolonial (Feldman 1975; Wichmann 2006; Becquey 2012). El chol es la lengua más difundida del 
subgrupo. Es hablada por más de 200 mil personas en el norte del estado de Chiapas, México. ${ }^{3}$ Según las estadísticas oficiales, alrededor de 38.500 personas hablan chontal en el estado de Tabasco en México, aunque la situación sociolingüística actual no favorece la vitalidad de la lengua (Delgado 2013: 10). La cantidad de hablantes del ch'orti' en el oriente de Guatemala se estima alrededor de 10 mil personas.

Las lenguas cholanas varían considerablemente en cuanto a la manera de expresar el significado prohibitivo. La sección 2.1 se dedica al chontal; se argumenta que esta lengua no tiene formas prohibitivas morfológica o sintácticamente específicas. La sección 2.2 describe los prohibitivos en ch'orti' que se forman por medio de partículas negativas específicas. La variedad de formas prohibitivas en chol se discute en la sección 2.3.

\subsection{Chontal de Tabasco}

En el chontal, la forma verbal que se utiliza con predicados transitivos para negar oraciones declarativas en el aspecto incompletivo también expresa el prohibitivo (Knowles-Berry 1987: 335-336). La entonación ayuda a resolver la ambigüedad (7).

Chontal

(7) mach a=jätz'-e'=on

NEG $\quad 2 \mathrm{~A}=$ golpear-INC=1B

'No me golpeas.' / ‘No me golpees!' (Knowles-Berry 1987: 335)

Los predicados intransitivos emplean el subjuntivo en construcciones prohibitivas (8).

Chontal

(8a) mach t'äb-ik=et

NEG subir-SUBJ=2B

'No subas.' (Knowles-Berry 1987: 343)

Los datos de la cantidad de hablantes aquí y adelante son de Law (2013: 143). 
(8b) mach nok-lek=et

NEG arrodillarse-SUBJ=2B

'No te pongas de rodillas.' (Keller y Luciano 1997: 465)

En efecto, los verbos transitivos nunca aceptan marcación del subjuntivo en chontal y utilizan el aspecto incompletivo en contextos donde los intransitivos toman los sufijos del subjuntivo (Vinogradov 2018a: 270). En ambos casos las formas prohibitivas no se distinguen morfológicamente de las formas declarativas negadas. Es decir, el chontal carece de la categoría gramatical del prohibitivo como tal. El significado correspondiente se expresa por la combinación de la partícula negativa general mach más el aspecto incompletivo o el modo subjuntivo, ${ }^{4}$ dependiendo de la transitividad del verbo.

Para expresar el imperativo (positivo), el chontal dispone de una serie de sufijos (9), y el alomorfo del imperativo depende de la clase del verbo; véase Osorio May (2016: 38, 43).

Chontal

(9a) chon-o

vender-IMP

‘Véndelo!' (Keller y Luciano 1997: 454)

(9b) t'äb-en

subir-IMP

‘Sube!’ (Knowles-Berry 1987: 343)

Las formas imperativas no se combinan con ningún otro aspecto ni modo (aunque algunas clases verbales toman sufijos en el imperativo, que son homónimos con los del incompletivo). Además, las formas imperativas carecen del marcador pronominal explícito, como en (9), aunque necesariamente hacen referencia a la segunda persona.

\footnotetext{
4 En chontal, el incompletivo y el subjuntivo son dos categorías del mismo paradigma de "tiempo/aspecto/modo" y, por lo tanto, son opuestas una a otra (véase Vinogradov 2018a). En otras palabras, no existen formas verbales marcadas por el incompletivo y el subjuntivo al mismo tiempo.
} 


\subsection{Ch'orti'}

Los prohibitivos en el ch'orti' hacen uso de una partícula negativa especial, ira (Schumann 2007: 239; Wichmann 1999: 46). Esta partícula precede a la forma verbal que no toma flexión de aspecto ni de modo (10).

Ch'orti'

(10a) ira i-cham-se

PROH 2PL.A-morir-CAUS

'No lo mates.' (Wichmann 1999: 46)

$\begin{array}{llllll}\text { (10b) ira i-t'ab'-ay } & \text { tama } & \text { e } & \text { te' } & \text { era } \\ \text { PROH } & \text { 2sG.C-subir-sUF } & \text { PREP } & \text { DEF } & \text { árbol } & \text { DEM } \\ \text { 'No subas a este árbol.' } & & & & \end{array}$

Schumann (2007: 239) también documenta la palabra ri'x 'ya no', que viene de la contracción de la partícula prohibitiva ira y el enclítico =i'x 'ya'. Dugan (2013) registra otra partícula negativa que, al igual que ira, se utiliza con prohibitivos: jola (11a). También aparece en los textos como joli, aparentemente ya llevando la marca de la segunda persona del juego pronominal C (11b). Hull (2016) afirma que la partícula jola es la variante dialectal de ira (176).

Ch'orti'

(11a) jol-

PROH-2SG.A vender 2SG.A-caballo

'No vendas tu caballo.'

$\begin{array}{llll}\text { jol-i } & \text { sut-pa } & \text { ta'-pat } & \text { aw-ira } \\ \text { PROH-2sG.C } & \text { regresar-MP } & \text { PREP.2sG.A-espalda } & \text { 2sG.A-ver }\end{array}$

'No voltees a ver.' (Dugan 2013: 228)

Es importante que las partículas prohibitivas ira y jola no se emplean en oraciones declarativas que se niegan con $m a^{\prime}$ y $m a^{\prime} c h i$ (12). 
Ch'orti'

(12a) e ixik era ma' b'anb'an

DEF mujer DEM NEG bueno

'Esta mujer no es buena.'

(12b) ma'chi $i<n>x i n$ in-cham-s-yet

NEG ir $<1$ SG.C $>$ 1SG.A-morir-CAUS-2sG.B

'No te voy a matar.'

Hay que hacer una breve observación sobre las formas del verbo que se utilizan en construcciones prohibitivas. A pesar de que no llevan marcación explícita de aspecto ni modo, la flexión pronominal que aparece en verbos intransitivos señala que se trata del aspecto incompletivo. Vinogradov (2018b: 169-170) describe el ch'orti' como una lengua excepcional dentro de la familia maya, que hace la distinción grammatical entre el completivo y el incompletivo solamente con verbos intransitivos, $\mathrm{y}$, además, lo hace mediante los marcadores de persona. Dependiendo del aspecto, se elige uno o el otro juego pronominal. En el completivo, la persona y número del único argumento del predicado intransitivo se indica por el juego B, mientras que para el incompletivo ha sido desarrollado el innovador juego pronominal C; véase también Robertson (1998). Los ejemplos (10b) y (11b) demuestran que en las construcciones prohibitivas aparecen los prefijos de la segunda persona del juego C, y no los enclíticos del juego B. Los verbos transitivos en ch'orti' no distinguen entre el completivo y el incompletivo; véase también del Moral (1988).

Los imperativos positivos se distinguen considerablemente de los prohibitivos en ch'orti'. Se utilizan sufijos específicos que dependen de la transitividad del verbo. Adicionalmente, igual que en chontal (la sección anterior), las formas imperativas no toman las marcas de persona (13).

Ch'orti'
(13a) kach-a
e tz'i'
amarrar-IMP DEF perro
'Amarra el perro.' 
(13b)
rajxa
regresar-MP-IMP pronto
'Regresa pronto.'

\subsection{Chol}

En cuanto a la expresión del significado prohibitivo, el chol parece ser la lengua más compleja del todo el subgrupo cholano. Esto tiene que ver tanto con una gran variación de formas prohibitivas como también con la variedad de estrategias de negar oraciones declarativas que complica el análisis de los prohibitivos. La manera más común de formar prohibitivos en chol (Coon 2006: 107; Vázquez Álvarez 2011: 294; Warkentin y Scott 1980: 48) es combinar la partícula negativa mach con la forma nominalizada del verbo (14).

Chol

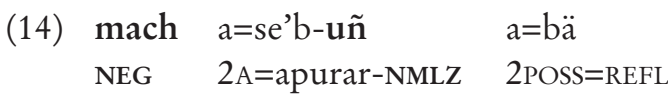

'No te apures.' (Selección 2001: 48)

La forma nominalizada del verbo principal que se ilustra en (14) es la misma forma que se utiliza en la lengua en el aspecto incompletivo (junto con un marcador específico del incompletivo que precede al verbo nominalizado), pero no aparece en el aspecto completivo; nótese la similitud con el ch'orti' (la sección anterior). Las construcciones prohibitivas opcionalmente llevan el enclítico $m e$, que desempeña una función ilocutiva (15).

Chol

$$
\begin{array}{ll}
\text { mach }=m e & a=\text { chok- } b-e \tilde{n} \\
\text { NEG }=I L L & 2 \mathrm{~A}=\text { tirar-APPL-NMLZ }
\end{array}
$$

'No se lo vayas a tirar.' (Meneses Méndez et al. 2013: 19)

Warkentin y Scott (1980: 48) describen otra manera de formar los prohibitivos en el chol. Se emplea la partícula negativa ma', más el marcador preverbal del tiempo/aspecto incompletivo mi como en 
(16). ${ }^{5}$ Según Warkentin y Scott, la diferencia entre dos formas del prohibitivo es dialectal: los prohibitivos con $m a$ ' se utilizan en Tila, mientras que el uso de mach caracteriza el dialecto de Tumbalá.

Chol

(16) ma' mi a=mäñ

NEG INC 2A=comprar.NMLZ

'No lo compres.' (Warkentin y Scott 1980: 48)

El análisis de textos publicados demuestra que también se puede utilizar el negador mach junto con la partícula del incompletivo mi (17), como también la forma $m a$ ' sin ningún marcador de tiempo/ aspecto (18).

Chol

(17a) mach mi a=tsäñ-s-añ=oñ

NEG INC $2 \mathrm{~A}=$ morir-CAUS-NMLZ=1B

'No me mates.' (Alarcón Estrada et al. 1998: 242)

(17b) mach mi la' jam

NEG INC 2PL.A abrir.NMLZ

'No lo abran (ustedes).' (Whittaker y Warkentin 1965: 128, \#69)

Chol

(18)

$$
\begin{array}{llll}
\mathrm{ma}=\mathrm{me}=\mathrm{ku} & \mathrm{a}=\mathrm{kan} & \text { aja } & \mathrm{aw}=\mathrm{uty}=\mathrm{i} \\
\mathrm{NEG}=\mathrm{ILL}=\mathrm{AFIRM} & 2 \mathrm{~A}=\mathrm{abrir} . \mathrm{NMLZ} & ? & 2 \mathrm{POSS}=\mathrm{ojo}=\mathrm{ENCL}
\end{array}
$$

'No abras los ojos.' (Alarcón Estrada et al. 1998: 227)

Se observa, pues, una gran variedad de estrategias. Las cuatro combinaciones de dos negadores y de formas con o sin marca del aspecto incompletivo son posibles, y la distribución no queda clara. Lo único que se puede afirmar con certeza es que la forma

5 La partícula del incompletivo mi y la marca de la segunda persona $a=$ a menudo se fusionan en $m a$ o $m a^{\prime}$. En (16) y (17a) se presentan las formas completas para evitar la confusión con la partícula negativa $m a^{\prime}$. 
prohibitiva con mach y sin marca aspectual, como en (14) y (15), es la que se encuentra con mayor frecuencia.

Entre los medios de negación de oraciones declarativas en chol se reportan dos partículas: mach y ma'añ. En cuanto a su distribución, Vázquez Álvarez dice que mach se utiliza con verbos sin marca prepositiva de aspecto y con predicados no verbales (menos los derivados de raíces posicionales), mientras que ma'añ se utiliza con verbos con marcadores de aspecto, con predicados posicionales y también para negar existencia de sustantivos (2011: 296). Coon argumenta que, cuando el hablante tiene posibilidad de escoger uno de los dos morfemas (por ejemplo, negando adjetivos), hay ciertas condiciones semánticas que influyen en la elección (2006: 102). Se plantea la idea de que mach niega predicados que expresan propiedades permanentes o esenciales, mientras ma'añ niega predicados que expresan propiedades temporales o transitorias. Para la discusión de prohibitivos, es importante que la posibilidad de negar formas declarativas en el incompletivo con la partícula mach está restringida a contextos muy específicos (19).

Chol

(19a) chukoch mach mi a=päy=oñ majl-el tyi paxyal ya' por.qué NEG INC $2 \mathrm{~A}=1$ lamar.NMLZ=1B ir-NMLZ PREP paseo allá tyi aw=otyoty

PREP 2POSS=casa

‘¿Por qué no me llevas a pasear a tu casa?' (Selección 2001: 48)

(19b) cha'añ mach mi laj=k=il-añ laj=wokol

para.que NEG INC PL.INCL=1A=ver-NMLZ 1PL.INCL.POSS=dificultad '...para que no tengamos problemas.' (Josserand y Hopkins 1996: 63)

La oración (19a) es una pregunta negativa que tiene ciertas consecuencias pragmáticas. Al hablante no le interesa tanto la respuesta directa, sino lo que quiere es causar que el oyente haga la acción sobre la cual le pregunta y que lo lleve a su casa. El ejemplo (19b) tampoco es neutral en cuanto a los propósitos extralingüísticos. Igual que el ejemplo anterior, implica una lectura modal. Cabe 
notar que los verbos en el aspecto incompletivo normalmente no se niegan en chol con mach ni con $m a^{\prime}$, sino con la palabra negativa ma'a ̃̃ (20).

Chol

(20)

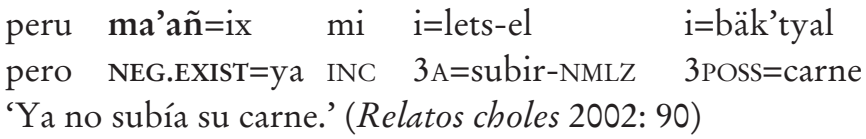

El negador $m a$ ' por sí solo no se utiliza en oraciones declarativas salvo el caso cuando sustituye a mach antes del clítico =ix ' $y a$ ' (21), aunque con otros enclíticos mach es perfectamente compatible.

Chol

$$
\begin{array}{lllc}
\text { ma'=ix } & \text { tyi } & \text { jul-i } & \text { iba } \\
\text { NEG=ya } & \text { COM } & \text { llegar-COM } & \text { 3sG } \\
\text { 'Él no llegó.' (Vázquez Álvarez } & \text { 2011: 362) }
\end{array}
$$

Hay que tener en cuenta que los ejemplos textuales, aunque lleven una traducción paralela, pueden ser interpretados de distintas maneras. Es probable que en algunos casos más bien se trate del uso de otra categoría con el significado secundario, y no de una forma prohibitiva como tal. Por ejemplo, las formas negadas en el aspecto incompletivo probablemente pueden interpretarse como una prohibición, al igual que el incompletivo positivo a veces puede ser entendido como una orden bajo ciertas circunstancias extralingüísticas (22).

Chol

mi $a=$ cha' bäk' sujty-el tyil-el INC $2 \mathrm{~A}=$ otra.vez luego regresar-NMLZ venir-NMLZ 'Regresa lo más pronto que puedas.' (Pérez López et al. 1994: 351)

Es probable, pues, que, en las ocasiones cuando después del negador mach aparece la marca mi del aspecto incompletivo, se 
trate del uso del incompletivo negativo en su función secundaria; compárese (17) y (19). Dado esta observación, se puede suponer que toda la variedad de construcciones prohibitivas en chol que hemos observado al inicio de esta sección puede ser reducida a dos estrategias generales. La primera estrategia consiste en usar la partícula $m a$; las construcciones de este tipo pueden opcionalmente involucrar la partícula del incompletivo mi. La otra estrategia es emplear la partícula negativa mach y no agregar ninguna marca de aspecto. Lo que difiere chol del ch'orti' (véase la sección 2.2) es que el uso de estas partículas negativas no se limita a las construcciones prohibitivas. Como hemos visto, aunque con ciertas restricciones, se utilizan en oraciones declarativas también. Por lo tanto, ni mach ni $m a$ ' pueden ser considerados como marcadores específicos del prohibitivo en chol.

Cabe notar que los imperativos positivos en chol tienen sus propios sufijos (que a veces coinciden con los sufijos de nominalización, según la clase del verbo). Además, los imperativos no llevan los marcadores de persona (23).

Chol

lets-en tyi k=paty $\quad \mathrm{y}=\mathrm{ik}$ 'oty muts'-u aw=uty subir-IMP PREP 1POSS=espalda 3POSS=con cerrar-IMP 2POSS=0jo 'Sube en mi espalda y cierra los ojos.' (Relatos choles 2002: 56)

Las formas imperativas no aceptan negadores y no pueden utilizarse en construcciones prohibitivas. Para formar "imperativos" de otras personas (es decir, exhortativos), se utilizan otras estrategias que involucran el modo subjuntivo.

\section{El significado prohibitivo en las lenguas tseltalanas}

El subrgupo tseltalano de la familia maya consta de dos lenguas: tseltal y tsotsil. Ambas lenguas son habladas en el sur de México por 445 mil y por 404 mil personas, respectivamente (Law 2013: 413). En las dos lenguas, el prohibitivo se forma mediante el prefijo 
$x^{-}$, que se reconstruye en el prototseltalano como el marcador del aspecto incompletivo (Robertson 1987). A continuación, la sección 3.1 describe las construcciones prohibitivas en tsotsil. Los datos del tseltal se presentan en la sección 3.2.

\subsection{Tsotsil}

La construcción prohibitiva en tsotsil se ilustra en (24). Los verbos, tanto intransitivos como transitivos, toman el prefijo $x-y$ se flexionan con los marcadores pronominales de la segunda persona, ya sea absolutivos o ergativos según la transitividad.

Tsotsil

(24a) $\mathbf{m u}=\mathrm{me} \quad \mathrm{x}$-a-xi'-ik
NEG=ILL $\quad$ INC-2B-temer-2PL
'No se espanten (ustedes).' (Laughlin 1977: 24$)$
(24b) $\mathbf{m u} \quad \mathbf{x}$-a-pas $\quad$ yech yan k'ak'al
NEG $\quad$ INC-2A-hacer así otro día
'No vuelvas a hacer eso.' (Laughlin 1977: 32)

El prefijo $x$ - se describe en las gramáticas como el "aspecto neutral” (Haviland 1981) o "timeless aspect” (Cowan 1969). Es el antiguo marcador del incompletivo lo que se corrobora por estudios diacrónicos que en la lengua moderna sigue desempeñando la misma función; véase Vinogradov (2016: 65-67). El sistema aspectual se complicó en el tsotsil moderno, ya que apareció un nuevo marcador del incompletivo: la partícula ta. Este morfema precede a las formas verbales que ya llevan el prefijo $x$-. Cuando ta no se separa del verbo por ningún enclítico, se observa una contracción, y esta combinación de morfemas se convierte en ch- (Delgaty 1961: 97; Haviland 1981: 109). Resulta que las tres maneras de marcar el aspecto incompletivo coexisten en tsotsil; compárese los ejemplos en (25).

Tsotsil

(25a) $x$-tal j-k'el=tik ta y-ox-ib-al k'ak'al 
INC-llegar 1A-ver=INCL PREP 3POSS-tres-NUM-ATTR día 'Llegaremos a verlo al tercer día.' (Laughlin 1977: 27)

(25b) ta $x$-k-ik' ech'-el li povre ants=e

INC INC-1A-tomar pasar-NMLZ DEF pobre mujer=ENCL

'Voy a sacar a la pobre mujer.' (Laughlin 1977: 45)

(25c) ch-i-muy ta ora ta krus

INC-1B-subir PREP hora PREP cruz

'Pronto voy a subir a la cruz.' (Laughlin 1977: 26)

Los tres marcadores no están en una distribución libre. Existen contextos en los cuales el prefijo $x$ - no puede ser sustituido por ninguno de sus alomorfos. Uno de estos contextos es el prohibitivo, que solamente acepta el morfema $x$-.

En cuanto a la negación, la partícula mu es la manera más simple (desde el punto de vista morfológico) de negar un predicado. En tsotsil existen también otros negadores: muk' y muk' bu. Laughlin (1975) explica la diferencia entre $m u$ y $m u k$ ' en términos de presencia/ausencia de voluntad o deseo por parte del hablante (241). En construcciones prohibitivas, se utiliza solamente la partícula negativa $m u$. Esto quiere decir que toda la variedad de negadores y marcadores del incompletivo se limita por una sola opción en las construcciones prohibitivas: la partícula $m u$ más el prefijo $x$-. Pero esta misma combinación se encuentra también en oraciones declarativas (26).

Tsotsil

(26a) k'u y-u'un mu $\quad$-av-ak' k-uch' vo'=e qué 3POss-causa NEG INC-2A-dar 1A-beber agua=ENCL ‘¿Por qué no me das de beber agua?’ (Laughlin 1977: 45)

(26b) $\mathbf{m u} \quad \mathrm{x}$-k-ak' a-vokol-ik

NEG INC-1A-dar 2POss-difícil-2PL

'No lo voy a hacer difícil para ustedes.' (Laughlin 1977: 48)

Igual que en las lenguas cholanas (la sección 2), el imperativo en tsotsil se forma de una manera muy distinta. Se utilizan los sufijos 
específicos, y la elección de alomorfos depende de la transitividad del verbo. Además, las formas imperativas no llevan las marcas de tiempo/aspecto ni de persona (27).

Tsotsil

$$
\begin{aligned}
& \text { tzak-o } \quad \text { uch’-an } \\
& \text { agarrar-IMP } \quad \text { beber-IMP } \\
& \text { ‘Agárralo! ¡Bebe!' (Laughlin 1977: 98) }
\end{aligned}
$$

Es interesante notar que en los textos se registran también otras formas del imperativo que son simétricas a las del prohibitivo. Hacen uso del mismo prefijo $x$ - (que aparentemente no es intercambiable con otros alomorfos del incompletivo), la marca de la segunda persona se mantiene, pero la partícula de negación $m u$ no aparece (28).

Tsotsil

$$
\begin{aligned}
& \text { (28a) } \mathbf{x} \text {-a-tal ik'-lum-an } \quad \mathbf{x} \text {-tal a-k'el } \\
& \text { INC-2B-venir negro-tierra-? INC-venir 2A-mirar } \\
& \text { 'Ven al amanecer, ven a ver.' (Laughlin 1977: 85) } \\
& \text { (28b) } \mathrm{x} \text {-a-ok' }=\text { me vo'ot } \quad \text { un }=e \\
& \text { INC-2B-cantar=ILL } 2 \mathrm{SG} \quad \text { pues }=\mathrm{ENCL} \\
& \text { 'Tú canta pues.' (Laughlin 1977: 27) }
\end{aligned}
$$

Se trata del uso secundario del incompletivo para denotar el imperativo, el mismo fenómeno que ya hemos discutido arriba en el chol (la sección 2.3). La función del prefijo $x$ - en este caso parece exceder el dominio semántico del aspecto incompletivo, llevando además ciertas implicaciones modales, lo cual no es sorprendente en vista de los datos del tseltal (véase la siguiente sección).

\subsection{Tseltal}

Para expresar el significado prohibitivo, el tseltal utiliza las formas verbales con el prefijo $x$ - (29). La estructura es, pues, similar a la del tsotsil. La partícula negativa en tseltal es ma. Igual que en tsotsil (la 
sección 3.1) y chol (la sección 2.3), el eclítico ilocutivo me en tseltal es opcional (Slocum 1980: 82).

Tseltal
(29a) $\mathbf{m a}=$ me $\mathbf{x}$-aw-uts'in=ix ta yan belta
NEG $=$ ILL PROH-2A-molestar=ya PREP otro vuelta
'Ya no lo molestes otra vez.' (Pérez López et al. 1994: 274)
(29b) $\mathbf{m a}=$ me $\quad \mathrm{x}$-yahl=at
NEG $=$ ILL PROH-caerse $=2 \mathrm{~B}$
'No te caigas.' (Slocum 1980: 83)

La diferencia entre el tseltal y el tsotsil concierne las funciones que el prefijo $x$ - desempeña fuera de las construcciones prohibitivas. Igual que en tsotsil, el prefijo $x$ - se utiliza en tseltal para indicar el incompletivo, pero solamente con verbos intransitivos. Otro marcador del incompletivo en tseltal es la partícula ya, que se emplea con cualquier tipo de verbo y coocurre con el prefijo $x$ - (30). A diferencia del prefijo $x$-, la partícula ya "puede ser omitida como señal de integración sintáctica o discursiva del verbo con lo que le precede" (Polian 2013: 162).

Tseltal
(30a) te
$\mathrm{a}-\mathrm{tat}=\mathrm{e}$
ya $\mathbf{x}$-tal
ta olil ajk'ubal
DEF 2POSS-padre=ENCL INC INC-venir PREP mitad noche
'Tu padre vendrá a la medianoche.'
(30b) pajel ya j-man chenek'
mañana INC 1A-comprar frijol
'Mañana compraré frijol.'

Con verbos intransitivos, el prefijo $x$ - provoca una ambigüedad: "las formas incompletivas negadas en segunda persona pueden interpretarse ya sea como formas indicativas, o como formas prohibitivas" (Polian 2013: 189). Con verbos transitivos, el prefijo $x$ - también se utiliza, pero desempeña otra función (31).

Tseltal 
(31a) sak

$\mathbf{x}$-a-bon te na=e

blanco EXH-2A-pintar DEF casa $=$ ENCL

'De blanco pintarás la casa.'

(31b) ti'bal=me $\mathbf{x}$-a-man

carne $=$ ILL $\quad$ EXH-2A-comprar

'Es carne que vas a comprar.' (Polian 2013: 200)

Polian caracteriza las formas ilustradas en (31) como "imperativas-exhortativas dependientes, que se usan en particular cuando el verbo viene después de un elemento focalizado, en particular un sustantivo, adverbio o demostrativo, o de predicado secundario" (2013: 199). Esta diferencia de significado implica que el prefijo $x$ por sí mismo no puede ser considerado como marca del prohibitivo en tseltal con verbos intransitivos ni transitivos.

En cuanto a la negación, el prohibitivo siempre utiliza el negador simple $m a($ '), y no utiliza el compuesto ma ba. Polian lo relaciona con el hecho de que la negación compuesta "corresponde a una menor implicación modal del hablante” (2013: 200).

Cabe notar que los predicados transitivos con el prefijo $x$ "pueden funcionar como imperativos por sí solos" en algunas variantes del tseltal (Polian 2013: 199). Es lo mismo que se observa en tsotsil ( $\sin$ tomar en cuenta la transitividad); véase los ejemplos (28) en la sección anterior.

\section{Datos complementarios del ch'olti'}

El ch'olti' es una lengua cholana extinta, lo que no permite obtener suficiente información sobre construcciones prohibitivas para un análisis exhaustivo. Sin embargo, es importante considerar los datos disponibles del ch'olti', ya que pueden complementar el panorama de las lenguas cholanas y corroborar las tendencias que se observan en las lenguas modernas.

El ch'olti' fue documentado en la época colonial en un solo manuscrito hecho a finales del siglo XVII (Kaufman y Norman 1984; Robertson 1998; Robertson et al. 2010). Este documento se atribuye al fray Francisco Morán y, por lo tanto, se refiere comúnmente como 
el “manuscrito de Morán”. El original se encuentra en los archivos de la Sociedad Filosófica Americana en Filadelfia (Law 2007; Robertson et al. 2010). La discusión en esta sección se basa principalmente en el análisis del manuscrito propuesto en Robertson et al. (2010).

Se identifican dos patrones generales de formar prohibitivos en el texto del manuscrito de Morán. El primer patrón utiliza el negador general ma más la forma verbal con el prefijo $x$ - que Robertson et al. (2010) analizan como la marca del tiempo futuro; véase también Kaufman y Norman (1984: 98). Aparentemente, al menos en contextos prohibitivos, este morfema tiene funciones modales. Algunos ejemplos de esta estrategia se presentan en (32).

Ch'olti'

$\begin{array}{lllll}\text { (32a) ma } & \text { ixte } & \mathbf{x} \text {-a-kale-n } & \text { xa } & \text { tahnal } \\ \text { NEG } & \text { entonces } & \text { FUT-2sG.A-hacer-FUT } & \text { de.nuevo } & \text { pecado }\end{array}$ 'Entonces no hagas pecado otra vez.' (Robertson et al. 2010: 94, \#420)

(32b) ma naik $\mathbf{x}$-aw-uta-n on tama u-kal-nah-el tahnal NEG ojalá FUT-2SG.A-guiar-FUT 1PL.B PREP 3A-hacer-PASS-NMLZ pecado 'Ojalá que no nos lleves a la tentación (lit., “al hacer pecado”).' (Robertson et al. 2010: 89, \#389)

(32c) ma x-a-bakl-e'n

NEG FUT-2sG.A-temer-1sG.B

'No me temas.' (Robertson et al. 2010: 99, \#459)

El otro patrón de formar prohibitivos es agregar un negador específico, el, a la forma verbal no marcada de aspecto, tiempo ni modo (33). El morfema el se caracteriza en Robertson et al. (2010: 183) como el "futuro negativo".

Ch'olti'

(33a) el a-muk-u

NEG.FUT 2sG.A-enterrar-SUF

'No los escondas.' (Robertson et al. 2010: 98, \#453) (33b) el aw-ubi ahxi'-il pehkahel 
NEG.FUT 2sG.A-escuchar maligno-? palabra

'No escuches palabras malignas.' (Robertson et al. 2010: 183, \#59b)

Las dos estrategias de formar prohibitivos en ch'olti' ya han sido observadas anteriormente en las lenguas modernas del subgrupo cholano-tseltalano. El uso de una partícula negativa específica más la forma verbal sin marcas de aspecto ni modo es exactamente el patrón que se da en ch'orti' (la sección 2.2). Aunque el morfema negativo particular puede ser distinto (no se observa una relación directa entre el en ch'olti' y joli ni ira en ch'orti'), la estructura general de las construcciones prohibitivas es igual. El uso del negador morfológicamente más simple y la forma verbal con el prefijo $x$ - se registra también en ambas lenguas tseltalanas, aunque en cada lengua individual este prefijo puede ser interpretado de distintas maneras, ya sea como un marcador aspectual, temporal o modal, dependiendo del sistema particular de categorías verbales.

Los datos del ch'olti' demuestran que dos distintos patrones de formar prohibitivos pueden coexistir. Además, tomando en cuenta que los dos patrones se registran en una lengua hablada en el siglo XVII, ninguno de ellos debe considerarse como innovador.

\section{Conclusiones}

Desde la perspectiva morfosintáctica, el prohibitivo es claramente distinto del imperativo en todas las lenguas cholanas y tseltalanas y no puede ser considerado como la contraparte negativa del imperativo. Hay tres diferencias principales entre el imperativo y el prohibitivo. Primero, las construcciones prohibitivas normalmente se forman a base de una categoría de tiempo (el futuro), aspecto (el incompletivo) o modo (el subjuntivo), y las construcciones imperativas no. Por lo tanto, el imperativo se considera como un modo particular, opuesto al modo indicativo (véase Vinogradov 2016), pero el prohibitivo pertenece al dominio del indicativo. La segunda diferencia concierne la marcación de persona. Las construcciones prohibitivas requieren de una marca explícita de la segunda persona 
(y es otro rasgo que comparten con las formas verbales en el modo indicativo), mientras que las construcciones imperativas no toman flexión de persona. El tercer argumento es puramente morfológico: algunos verbos tienen formas imperativas supletivas, como en (34), pero los prohibitivos supletivos no existen.

Chol

(34a) kuku säkl-añ ya' baki mi a=majl-el tyi wäy-el ir.IMP buscar-NMLZ allá donde INC 2A=ir-NMLZ PREP dormir-NMLZ 'Ve a buscar adónde irás a dormir.' (Selección 2001: 65)
la' $=\mathrm{ix}=\mathrm{ku}$
che' woli tyi oñ-el
ili lak=ña'
venir.IMP=ya=AFIRM así PROG PREP gritar-NMLZ DEM 1PL.INCL. POss-madre

‘Ven ya! - gritaba la señora.’ (Selección 2001: 76)

Esto quiere decir que el prohibitivo no es simétrico al imperativo en ninguna lengua del subgrupo cholano-tseltalano de la familia maya. La única semejanza entre los imperativos y los prohibitivos concierne al uso del enclítico me (al menos, en chol y en las lenguas tseltalanas). Este morfema indica cierta fuerza ilocutiva que caracteriza tanto oraciones imperativas como prohibitivas.

Las diferencias entre los prohibitivos y los declarativos negativos no son tan sobresalientes y varían considerablemente en las lenguas individuales. En total, en cinco lenguas se observan tres tipos de relación entre el prohibitivo y el declarativo negativo (véase la figura 1).

El chontal no tiene prohibitivo específico y utiliza formas declarativas negativas en el incompletivo o subjuntivo, dependiendo de la transitividad del verbo. La desambiguación se lleva a cabo por medios no gramaticales, como la entonación, por ejemplo. Ch'orti', en cambio, dispone de un negador específico cuyo uso está restringido a los prohibitivos. Por lo tanto, en ch'orti' no hay ambigüedad entre el prohibitivo y el declarativo negativo. El tercer tipo de relación se puede denominar como "híbrido"; es el más complejo para describir y analizar. Se observa en chol, tsotsil y tseltal. 
Figura 1. Los patrones de formación de prohibitivos en las lenguas cholano-tseltalanas

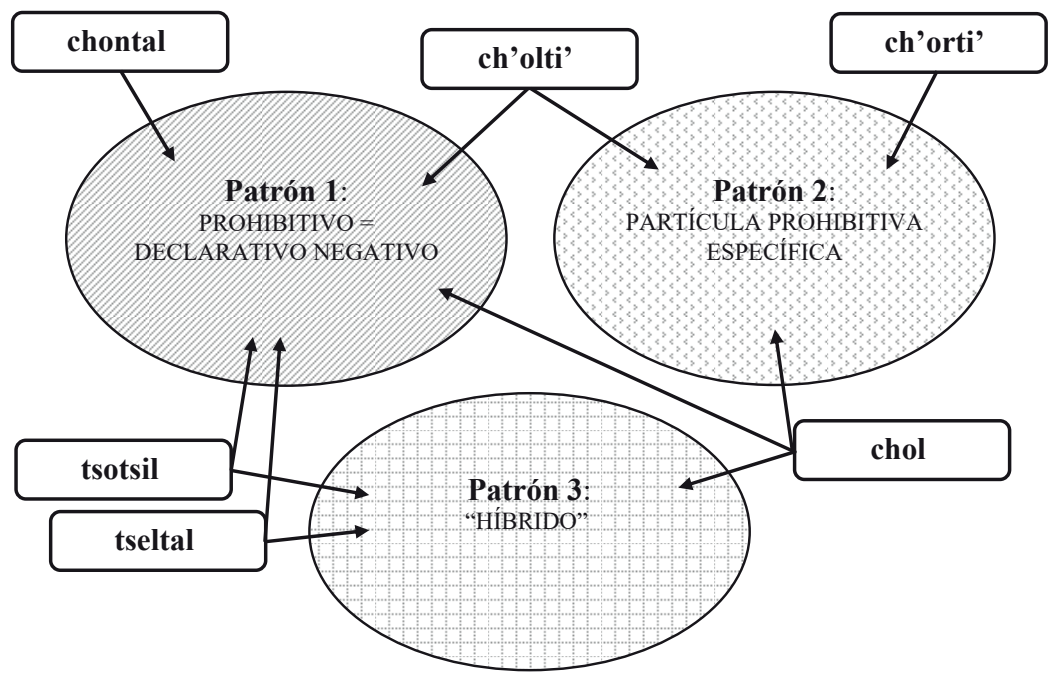

En estas lenguas, en algunas ocasiones las formas verbales del prohibitivo y del incompletivo negativo son ambiguas, pero en otras son diferentes, aunque ningún morfema puede considerarse como el marcador dedicado específicamente a denotar el significado prohibitivo. En tsotsil, por ejemplo, la elección de la marca del incompletivo en construcciones prohibitivas se restringue a un único alomorfo: el prefijo $x$-. En tseltal, el prefijo $x$ - que aparece en construcciones prohibitivas se utiliza para indicar el incompletivo solamente con verbos intransitivos. En cuanto a la negación, se utiliza el marcador que es morfológicamente el más simple y, al mismo tiempo, que implica mayor interés por parte del hablante.

Como hemos dicho en la introducción, los datos comparativos de lenguas emparentadas permiten plantear hipótesis acerca del desarrollo diacrónico. El presente estudio corrobora la idea que el prefijo $x$ - en tsotsil y tseltal era antiguamente el marcador general del incompletivo; véase las consideraciones diacrónicas en 
Polian (2013: 161) y Robertson (1987: 440). En tsotsil moderno, a su base se desarrollaron otras marcas del incompletivo, y en tseltal, su significado fue expandido al dominio modal. Es probable que tres estrategias descritas arriba en el nivel sincrónico en realidad provengan de solo dos estrategias que existían antiguamente. La primera es emplear una partícula negativa específica. Esta estrategia se mantiene en el ch'orti' y se documenta para el ch'olti'. La segunda es negar oraciones declarativas, ya sea en el modo subjuntivo o, con mayor frecuencia, en el aspecto incompletivo (que en algunas lenguas mayas también puede implicar una lectura modal; véase Vinogradov 2014). Esta estrategia se mantiene intacta en chontal de Tabasco y engendró las estrategias "híbridas" en chol, tsotsil y tseltal.

El patrón "híbrido" aparece por causa de la reestructuración diacrónica de sistemas verbales. En particular, se debe al desarrollo de múltiples maneras de negar oraciones declarativas (en chol) y a los cambios morfológicos en la marcación del aspecto incompletivo (en tsotsil y tseltal). Las formas prohibitivas demuestran la etapa diacrónica más antigua, lo que se corrobora, por ejemplo, por el hecho de que utilizan los negadores morfológicamente más simples que sirvieron como base para formar negadores nuevos. La situación en el chol es particularmente interesante ya que los cambios en el sistema de negación resultaron en que el negador general $m a$ ' fue sustituido por las formas más complejas mach y ma'añ en oraciones declarativas, pero se mantuvo en (algunas) oraciones prohibitivas. De esta manera la partícula $m a^{\prime}$ en chol llegó a parecer a los negadores prohibitivos específicos como los que se observan en ch'orti' y ch'olti'. Sin embargo, en el caso del chol, este patrón de formación de prohibitivos no proviene del estado de lengua más antiguo, sino representa una innovación en el sistema gramatical. 


\section{Referencias bibliográficas}

ANÓNIMO

2001 Selección de cuentos: chol. Tuxtla Gutiérrez: Gobierno del Estado de Chiapas.

ANÓNIMO

2002

Relatos choles. México D.F.: Dirección General de Culturas Populares e Indígenas.

Aikhenvald, Alexandra

2010 Imperatives and Commands. Oxford: Oxford University Press.

Alarcón Estrada, Verónica, Víctor Esponda Jimeno y Antonio Gómez GÓMEZ (eds.)

1998 Cuentos y relatos indígenas, tomo VII. San Cristóbal de las Casas: Universidad Nacional Autónoma de México/Universidad Autónoma de Chiapas.

Becquey, Cédric

2012 "Quelles frontières pour les populations cholanes ?" Ateliers d'Anthropologie. 37. Consultado: 10 de mayo de 2018. $<$ https://journals.openedition.org/ateliers/9181>.

BISANG, Walter

2004 "Dialectology and Typology - An Integrative Perspective". En Dialectology Meets Typology: Dialect Grammar from a Cross-Linguistic Perspective. Ed., Bernd Kortmann. Berlin y Nueva York: Mouton de Gruyter, 11-45.

Chamoreau, Claudine

2012 "Dialectology, Typology, Diachrony and Contact Linguistics: A Multi-Layered Perspective in Purepecha". STUF-Language Typology and Universals. 65, 1, 6-25.

Coon, Jessica

2006 "Existentials and Negation in Chol (Mayan)". En CamLing: Proceedings of the Fourth University of Cambridge Conference in Language Research . Eds., Charles Chang, Esuna Dugarova, Irene Theodoropoulou, Elina Vilar Beltrán y Edward Wilford. Cambridge: Cambridge Institute of Language Research, 51-58. 
Cowan, Marion

1969 Tzotzil Grammar. Norman: Summer Institute of Linguistics.

Del Moral, Raúl

1988 "Introducción al sistema verbal del chortí de Guatemala". Estudios de Cultura Maya. XVII, 397-421.

Delgado Galván, Amanda

2013 “Topological Expressions in Yokot'an (Chontal de Tabasco), Nacajuca Dialect”. Tesis de Maestría. Universidad de Leiden.

Delgaty, Colin

1961 “Tzotzil Verb Phrase Structure”. En Mayan Studies, Vol.I.Ed., Benjamin Olson. Norman: Summer Institute of Linguistics, $83-126$.

Dugan, James Timothy

2013 “The grammar of Ch'orti' Maya folktales". Tesis de Doctorado. Universidad Tulane.

FeLdman, Lawrence

1975 Riverine Maya: The Torquegua and Other Chols of the Lower Motagua Valley. Columbia: University of Missouri.

Forker, Diana

2013 "Microtypology and the Tsezic Languages: A Case Study of Syntactic Properties of Converbal Clauses". SKY Journal of Linguistics. 26, 21-40.

Greenberg, Joseph H.

1969 Some Methods of Dynamic Comparison in Linguistics. En Substance and Structure of Language. Ed., Jaan Puhvel. Berkeley: University of California Press, 147-203.

Haviland, John B.

1981 Sk'op sotz'leb - el tzotzil de San Lorenzo Zinacantán. México D.F.: Universidad Nacional Autónoma de México.

Hull, Kerry

2016 A Dictionary of Ch'orti' Mayan-Spanish-English. Salt Lake City: University of Utah Press.

Josserand, Kathryn y Nicholas A. Hopkins

1996 Chol Ritual Language. FAMSI Research Report. Consultado: 9 de abril de 2018. <http://www.famsi.org/reports/94017/index. html>. 
Kaufman, Terrence y William Norman

1984 "An Outline of Proto-Cholan Phonology, Morphology, and Vocabulary". En Phoneticism in Mayan Hieroglyphic Writing. Eds., John Justeson y Lyle Campbell. Albany: State University of New York, 77-166.

Keller, Kathryn y Plácido LuCiano

1997 Diccionario chontal de Tabasco (mayense). Tucson: Summer Institute of Linguistics. Consultado: 3 de abril de 2018. <www. mexico.sil.org/resources/archives/10973>.

KIBRIK, Aleksandr E.

1998 “Does Intragenetic Typology Make Sense?” En Sprache im Raum und Zeit: In Memoriam Johannes Bechert, Band 2. Eds., Winfried Boeder, Christoph Schroeder, Karl Heinz Wagner y Wolfgang Wildgen. Tübingen: Narr, 61-68.

KNOWLES-BERRY, Susan

1987 "Negation in Chontal Mayan". International Journal of American Linguistics. 53, 3, 327-347.

LaUghlin, Robert M.

1975 The Great Tzotzil Dictionary of San Lorenzo Zinacantán. Smithsonian Contributions to Anthropology, 19. Washington D.C.: Smithsonian Institution Press.

1977 Of Cabbages and Kings: Tales from Zinacantán. Smithsonian Contributions to Anthropology, 23. Washington D.C.: Smithsonian Institution Press.

LAw, Danny

2007 "Poetic Style in Colonial Ch'olti' Mayan". Latin American Indian Literatures Journal. 23, 2, 142-168.

2013 "Mayan Historical Linguistics in a New Age". Language and Linguistics Compass. 7, 3, 141-156. https://doi.org/10.1111/ $\operatorname{lnc} 3.12012$

Meneses Méndez, Domingo, Omar López Espinoza y Carlos Ignacio López Bravo

2013 Compilación de cuentos chiapanecos. México D.F.: Terracota. Consultado: 9 de abril de 2018. <http://www.unich.edu.mx/ libros-gratuitos/6144>. 
Miestamo, Matti y Johan Van Der Auwera

2007 "Negative Declaratives and Negative Imperatives: Similarities and Differences". En Linguistics Festival, May 2006, Bremen. Ed., Andreas Ammann. Bochum: Brockmeyer, 59-78.

Mó IsÉm, Romelia

2006 "Fonología y morfología del Poqomchi' occidental". Tesis de Licenciatura. Universidad Rafael Landívar, Guatemala.

Osorio May, José del Carmen

2016 Temas de la sintaxis del yokot'an "chontal" de Tecoluta, Nacajuca, Tabasco. Tesis de Doctorado. Centro de Investigaciones y Estudios Superiores en Antropología Social, México.

Pérez López, Enrique, Manuel Hidalgo Pérez y Antonio Gómez Gómez (eds.)

1994 Cuentos y relatos indígenas, tomo V. México D.F.: Universidad Nacional Autónoma de México.

Polian, Gilles

2013 Gramática del tseltal de Oxchuc. México D.F.: Centro de Investigaciones y Estudios Superiores en Antropología Social.

POLINSKY, Maria

2010 "Linguistic Typology and Formal Grammar". En The Oxford Handbook of Typology. Ed., Jae Jung Sung. Oxford: Oxford University Press, 650-665. https://doi.org/10.1093/ oxfordhb/9780199281251.013.0031

ROBERTSON, John S.

1987 "The Common Beginning and Evolution of the Tense-Aspect System of Tzotzil and Tzeltal Mayan". International Journal of American Linguistics. 53, 4, 423-444.

1998 "A Ch'olti'an Explanation for Ch'orti'an Grammar: A Postlude to the Language of the Classic Maya”. Mayab. 11, 5-11.

Robertson, John S., Danny Law y Robbie A. Haertel

2010 Colonial Ch'olti': The Seventeenth-Century Morán Manuscript. Norman: University of Oklahoma Press.

Romero, Sergio

2012 "A Maya Version of Jespersen's Cycle: The Diachronic Evolution of Negative Markers in K'iche' Maya”. International Journal of American Linguistics. 78, 1, 77-96. 
Schumann Gálvez, Otto

2007 Introducción a la morfología verbal del chortí. Graz: Academic Publishers.

SHLuinsky, Andrey

2017 “An Intragenetic Typology of Kwa Serial Verb Constructions”.

Linguistic Typology. 21, 2, 333-385. https://doi.org/10.1515/ lingty-2017-0008

Slocum, Marianna

1980 “A Sketch of Bachajon Tzeltal Clause and Sentence Structure”. SIL-Mexico Workpapers. 3, 79-92.

VAn Der Auwera, Johan

2006 "Why Languages Prefer Prohibitives?" Journal of Foreign Languages. 2006, 1, 2-25.

2010 "Prohibition: Constructions and Markers". En Contrasting Meaning in Languages of the East and the West. Eds., Dingfang Shu y Ken Turner. Berna: Lang, 443-475.

Van Der Auwera, Johan y Maud Devos

2012 "Irrealis in Positive Imperatives and in Prohibitives". Language Sciences. 34, 2, 171-183. https://doi.org/10.1016/ j.langsci.2011.08.003

Van Der Auwera, Johan, Ludo Lejeune y Valentin Goussev

2005 “The Prohibitive”. En The World Atlas of Language tructures. Eds., Martin Haspelmath, Matthew Dryer, David Gil y Bernard Comrie. Oxford: Oxford University Press, 290-293.

VÁzQuez Álvarez, Juan Jesús

2011 “A Grammar of Chol, a Mayan Language”. Tesis de Doctorado. Universidad de Texas en Austin.

VINOGRADOV, Igor

2014 La categoría de incompletivo en las lenguas mayas: un estudio comparativo de variación semántica. Studii de gramatică contrastivă. 21, 65-82.

2016 Tense/Aspect/Mood Systems of Cholan-Tseltalan Mayan Languages. Múnich: Lincom Europa.

2018a “Grammatical Periphery of Chontal Maya Verb”. University of British Columbia Working Papers in Linguistics. 46, 266-280. 
2018b "Los sistemas de tiempo/aspecto/modo yucatecanos y cholanos: semejanzas y diferencias". Cuadernos de Lingüística de El Colegio de México. 5, 1, 148-182.

Von WaLdenfels, Ruprecht

2014 "Explorations into Variation across Slavic: Taking a BottomUp Approach". En Aggregating Dialectology, Typology, and Register Analysis: Linguistic Variation in Text and Speech. Eds., Benedikt Szmrecsanyi y Bernhard Wälchli. Berlin y Boston: Walter de Gruyter, 290-323.

Warkentin, Viola y Ruby SCOTT

1980 Gramática ch'ol. México D.F.: Instituto Lingüístico de Verano. Consultado: 3 de abril de 2018. <http://www.mexico. sil.org/resources/archives/1699>.

WhitTAKer, Arabelle y Viola Warkentin

1965 Chol texts on the supernatural. Norman: Summer Institute of Linguistics.

WiChMANN, Søren

1999 A Ch'orti' Morphological Sketch. Unpublished manuscript.

2006 "Mayan Historical Linguistics and Epigraphy: A New Synthesis". Annual Review of Anthropology. 35, 279-294.

Recepción: 21/12/2018

Aceptación: 09/10/2019 